\title{
El papel de la Universidad Pública en el bienestar social y en el derecho al trabajo*
}

\section{Carlos Javier Martínez G.}

Magister en desarrollo educativo y social, docente facultad de derecho Universidad

\section{Resumen}

La disertación desarrolla tres aspectos. Primero, una reflexión teórica sobre los efectos de la actual crisis estructural de las universidades públicas y el bienestar social; segundo, presenta, con base en algunas cifras, las magnitudes del subdesarrollo en América Latina y el Caribe y su influencia en la ciencia, la tecnología, la educación, el trabajo y, en consecuencia, el bienestar individual y social. Por último, expone las bases teóricas e históricas que han llevado a la Facultad de Derecho de la Universidad Surcolombiana, a proponer la creación de la Especialización en Derecho del Trabajo y la Seguridad Social, cuya aprobación se espera cumplir próximamente.

\section{Palabras claves}

Crisis, Universidad Pública, Bienestar Social, Subdesarrollo, Trabajo. Educación.

\section{Abstract}

The rol of the public university in social well-Jare and in the labor law

The essay develops three topics: First, a theoretical reflection about the effects of the current structural crisis of the public universities and the social well-fare; second, based on some numbers, it presents the magnitude of sub development in Latin America and The Caribbean and its influence in Science, Technology, Education, Labor and, consequently, the individual and social well-fare. Finally, it exposes the theoretical and historical bases that had carried the Low Faculty of the Universidad Surcolombiana to propose the creation of the Specialization on Labor and Social Security Law, which approbation is nearly expected.

\footnotetext{
* Ponencia presentada en el Foro sobre la Protección de los Derechos Fundamentales del Trabajo, realizado por el Ministerio de la Protección Social y la OIT, a través de la Dirección Territorial del Huila, Neiva, julio de 2004.
} 
Hoy se asume un nuevo tipo de sociedad en la que lo único le da sentido a esta es el mercado sus regulaciones: así, está surgiendo un nuevo sujeto sin derecho que no reconoce el derecho del otro y atenta contra él, porque es negado por la sociedad.

Han surgido, por desgracia, nuevos actores sociales que solo se guían por el presente, sin importar el pasado ni el futuro.

En este contexto adquiere un papel fundamental la Universidad Pública en cuanto cumpla con su función social e intelectual en la conformación de una 'región inteligente', es decir, una región capaz de analizar los problemas relevantes que le competen y proponer soluciones viables a estos problemas.

La incorporación de la Universidad al debate provoca la necesidad apremiante de una reflexión a la luz de los nuevos tiempos que transcurren, ya que de marchar la sociedad como estaba previsto antes de esta profunda crisis estructural, las universidades se habrían convertido inevitablemente en centros privilegiados de producción y difusión del saber transformador universal, donde el conocimiento ocuparía un lugar preponderante. Ahora, nadie cree que la Universidad vaya a conducir a algún cambio revolucionario; por el contrario, hay fuertes dudas de su participación en el proceso de modernización, olvidando que la crisis tiene un objetivo central, aunque no explícito: liquidar la posibilidad de la Educación Superior Estatal y, con ello, impedir que la universidad pública se consolide y desarrolle como la única vía que tienen vastos sectores de la población para acceder a la formación y al conocimiento de nivel superior que les permita un mejor vivir, un trabajo más digno, una retribución equitativa y la posibilidad de disentir sin temor a ser castigado por ello.

Esta problemática ha llevado a que las universidades públicas vayan perdiendo gran parte del sitio que ocupaban en el seno de la sociedad, la industria, el comercio y los espacios de discusión y decisión política. Así, no debe sorprendernos que el sistema de enseñanza actual sea tan «racional» que está pensado más en función de las materias que ha de dominar el estudiante, que en función del desarrollo del individuo y de la sociedad. Tampoco debe sorprendernos que la universidad sea mal calificada en cuanto a sus aportes a la solución de problemas similares a los que hoy nos convocan a la reflexión y a la acción, se le discute a la universidad su poco aporte a lo social', aún sin tener en claro a qué categoría se refiere lo social'.

En parte, aquí radica precisamente la esencia del problema actual de cómo enfrentan las Universidades Públicas esa pérdida de su espacio vital, qué retos tienen que vencer y cómo van a dar solución a cada uno de ellos. 
Es necesario, por tanto, que las Instituciones Públicas de Educación Superior estén a disposición de los gobiernos, como agentes activos que formulan e implementan la política en general, y en particular las políticas de educación, ciencia y tecnología relacionadas con la educación, la salud y el trabajo. En este contexto, abordar la problemática actual de la sociedad de manera conjunta por los académicos, los políticos, los industriales y los comerciantes no constituye un simple ejercicio académico, sino una realidad impositiva por cuanto hoy en día la integración, las finanzas y la información están creando una cultura y un mercado global único, que Friedman llama «un tren sin freno».

Tal y como se afirma en muchos estudios y estudiosos de las relaciones entre la universidad y el bienestar de la sociedad, las universidades públicas son piezas básicas de nuestra sociedad que requieren dinamismo y flexibilidad para adaptarse -y adelantarse, en lo posible- a los cambios que nos rodean. Necesitamos universidades públicas dotadas de un buen sistema de gobierno y con los medios suficientes para crear, producir y difundir el conocimiento; universidades para formar hoy y mañana de manera permanente a nuestros hombres y mujeres, con el fin de que puedan desarrollar una tarea que les permita conseguir una calidad de vida digna, dentro del marco de una sociedad justa y de progreso.

Hoy, la Universidad Pública tiene que cumplir su función crítica dentro de la sociedad y debe cumplir su misión pública -el desarrollo de la educación ciudadana- para poder enfrentarse a los desafíos actuales de la Globalización y la Glocalización, pues se trata, en síntesis, de pensar de modo global, pero de actuar de modo local. El gran desafío que tienen los académicos es la decisión de adoptar una postura ante las problemáticas sociales, adoptar nuevas formas de pensar y de actuar en el cambiante entorno conformado por un mundo cada vez más complejo y proponer nuevos modelos para generar un sentido de la vida que redunde en mejores niveles de bienestar para todos.

\section{Segundo}

Es interesante estudiar las relaciones fuertes que existen entre el conocimiento, la investigación y el desarrollo social y económico, apoyados en algunas cifras sobre la situación en Colombia en estos aspectos y su relación con otros países del área, para que se tenga una idea aproximada de la magnitud del problema que vive el país en este aspecto. En particular, lo vemos interesante si aceptamos que las oportunidades de acceso al trabajo, la calidad del mismo y la protección de sus derechos fundamentales, están en relación directa con los índices de desarrollo humano, social, participativo y académico de una sociedad.

Nos han vendido la idea de que Colombia está a la vanguardia en estos asuntos, 
es más, que somos un país rico y por ello nos refriegan a cada rato el cuento de que cómo es posible que siendo Colombia un verdadero jardín, es más un edén, tenga los problemas que tiene, ocultando que la magnitud de los problemas, es más compleja de lo que parece.

Confirmemos estas apreciaciones, con base en algunos datos sobre la Ciencia, en general; sobre la situación de Colombia en relación con los países de América Latina y la situación de la Educación Superior en el país. Lo que se pretende es tener una idea global de cómo estamos en estos aspectos en comparación con países del área, no con países de mayor tradición y desarrollo en estos campos.

Si el siglo XX fue el de la microelectrónica, que marcó el gran avance en el desarrollo científico-tecnológico que revolucionó todas nuestras formas y medios de producción, el presente siglo será el de la Nanoelectrónica, que multiplicará por un millón la capacidad de almacenamiento de datos y reducirá en una proporción similar a un millón, los espacios necesarios para almacenar la misma cantidad de datos. Este fenómeno 'reduccionista' tendrá serias implicaciones laborales, económicas y jurídicas cuyas dimensiones todavía no podemos prever. Es lógico predecir, desde esta óptica, el tipo, de actividades industriales, comerciales y de servicios que tendrán mayores posibilidades de éxito, en el mundo, dentro de un marco de economía globalizada. Dentro de estas tendencias podríamos señalar:

$>$ Cura definitiva de enfermedades o mayor alcance de expectativa de vida.

$>$ Desarrollo de superinteligencias, ya sea de modo artificial o mediante manipulación genética de seres humanos.

> Producción ilimitada de energía.

> La producción de seres vivos, que es cada día una realidad más palpable.

Por todo lo anterior, es evidente que estamos en la llamada 'sociedad del conocimiento'; pero también es evidente que a mayor conocimiento existe mayor riesgo, desde cualquier ángulo que se le mire. Baste con considerar que en todas las áreas del conocimiento la información se duplicará cada seis meses, en los próximos diez años, lo que nos está condenando a ser analfabetas constantes y a buscar, a veces con desespero, oportunidades de capacitación cada vez más específicas para el desempeño de funciones cada vez más específicas también, segmentando las posibilidades del conocimiento a una sola de sus aristas y representando un reto enorme reflejado en la super especialización, en desmedro de la comprensión global del mundo y sus fenómenos. Sus implicaciones en el derecho del trabajo serán cada vez mayores: cuando el $95 \%$ de nuestra población no tiene acceso a la formación adecuada, esta población se verá obligada a aceptar condiciones laborales que riñen con los derechos fundamentales. 
Bajo este marco global de referencia, veamos algunas cifras sobre la situación en América Latina, que pueden ilustrarnos sobre el papel que cumplimos en el concierto regional y mundial.

Para la región, encontramos algunos datos interesantes en relación con el año 2002 y sus proyecciones para el 2003, en lo relacionado con el PIB real, la deuda externa y el desempleo, así:

\section{PIB Real (en \% de cambio)}

\begin{tabular}{lrr} 
PAÍS & 2002 & 2003 \\
\hline BRASIL & 1.4 & 2 \\
ECUADOR & 2.6 & 3.2 \\
MÉXICO & 1.1 & 2.7 \\
COLOMBIA & 1.5 & 2.5 \\
VENEZUELA & -8.2 & -7.2 \\
PERÚ & 4.9 & 4.5 \\
CHILE & 2.8 & 3.2 \\
COSTA RICA & 2.8 & 3.0 \\
URUGUAY & -10.5 & -0.5 \\
ARGENTINA & -11.7 & 2.0
\end{tabular}

Deuda externa (en US\$ miles de Millones)

\begin{tabular}{lrr} 
PAÍS & 2002 & 2003 \\
\hline BRASIL & 210 & 215 \\
ECUADOR & 13.8 & 16.9 \\
MÉXICO & 161 & 164 \\
COLOMBIA & 40.8 & 42.3 \\
VENEZUELA & 22.3 & 32.5
\end{tabular}

\section{Desempleo (año 2002)}

\begin{tabular}{lr} 
PAÍS & $\%$ \\
\hline BRASIL & 11.7 \\
ECUADOR & 8.4 \\
MÉXICO & 2.6 \\
COLOMBIA & 15.6 \\
VENEZUELA & 18.0 \\
PERÚ & 8.7 \\
CHILE & 7.8 \\
COSTA RICA & 6.4 \\
URUGUAY & 18.6 \\
ARGENTINA & 16.8
\end{tabular}


Como puede ser visto, estos indicadores económicos no son los más alentadores y la posición de Colombia no es muy cómoda, lo que incide, y de qué manera, en el apoyo real a los procesos de investigación, desarrollo científico y educación y, por ende, en el derecho a un trabajo digno, con remuneración equitativa y sin discriminaciones artificiosas.

Como simple comparación, veamos algunos indicadores de América Latina y de los países desarrollados.

La región representa cerca del $8 \%$ de la población mundial y aporta el 7\% del Producto Interno Bruto; además, produce el $6 \%$ de los bienes manufacturados y el $3 \%$ de los bienes de capital. Como puede ser visto, su impacto económico no es muy grande y ello podría explicar la atención de soslayo que casi siempre le han puesto a sus problemas la gran mayoría de los países superdesarollados.

El desbalance de la región, comienza a ser palpable, si anotamos que sólo cuenta con el $2.4 \%$ de los científicos del mundo, mientras que Europa tiene el 22\%, los Estados Unidos el 19\% y Asia, otro 19\%. El resto, está distribuidos entre África y Oceanía.

Para Investigación y Desarrollo se gastan aproximadamente 10.000.000 de millones de dólares anuales, en todo el mundo, y América Latina participa con apenas el $1.8 \%$ de esta cifra, mientras Europa aporta el 34\%, Norteamérica el $33 \%$ y Asia el 24\%. Aquí, el desequilibrio es dramático puesto que la cifra de participación ni siquiera es similar al de la población y las diferencias entre esta región y las otras son abismales. Para completar el panorama, Colombia escasamente participa con el $0.7 \%$ de lo correspondiente a América Latina.

Por tanto, no debe sorprendernos que nuestro país ocupe puestos intermedios, e incluso inferiores, en cuanto a Investigación Aplicada.

En consecuencia, decir que Colombia es un país fuerte, rico, de amplias potencialidades, con muchos recursos, maravilloso y todos esos eufemismos con los que nos llena el discurso oficial que pretende tapar el sol con un dedo, no pasa de ser un sofisma aberrante de ocultamiento de una problemática explosiva, tal y como se ve en la actualidad. En otras palabras, parodiando a Galeano cuando se refería a la situación de España durante la Conquista y la Colonia, puede ser que «Colombia tenga la vaca, pero otros se toman la leche».

Respecto a la educación en Colombia, podemos señalar que, el presupuesto para educación se ha mantenido alrededor del $3.07 \%$ del PIB, y para Ciencia y Tecnología apenas del $0.02 \%$ de este PIB. Con esta cantidad es imposible pensar en un verdadero desarrollo y el cuento de que somos un país en 'vía de 
desarrollo', no pasa de ser una perversión mental y lingüística para no decirnos directamente 'subdesarrollados'.

La matrícula en el Sistema de Educación Superior llegó a aproximadamente 900.000 alumnos, de los cuales 300.000 pertenecen al sector público (el 33.3\%) y 600.000, al sector privado (el 66.7\%), en otras palabras, de cada tres estudiantes, uno pertenece al sector público, a pesar de que la educación es un servicio público.

Los Programas Académicos con mayor demanda son los del área de las Ciencias Económicas y Administrativas, que tienen una matrícula total de cerca de 135.000 alumnos (14.8\%), a pesar de que se cuenta con más de 4.000 programas legalmente registrados ante el Ministerio de Educación Nacional.

Existen 101 universidades, 84 instituciones universitarias, 59 institutos tecnológicos, 1 escuela tecnológica, 52 de formación técnica, 14 de régimen especial, para un total de 311 centros de Educación Superior; de ellos, 210, es decir el $67.5 \%$, son de carácter privado. Si bien el problema básico no es la naturaleza de la institución, sí lo constituye el hecho de que muchas de estas instituciones no cumplen con requisitos mínimos que las acrediten como tales y sus aportes al desarrollo científico, tecnológico $\mathrm{y}$ al conocimiento $\mathrm{y}$ empoderamiento de la sociedad son muy cuestionables.

En el nivel postuniversitario, donde se supone que se deben generar los aportes teóricos, metodológicos y técnicos para el verdadero desarrollo de un país, se tiene una matrícula total de 46.000 alumnos, de los cuales el $90 \%$ están en cursos de Especialización (41.400), el 9.5\%, en cursos de Maestría (4.370) y apenas el 0.4, en Doctorado (230). Existen algunas personas cursando su doctorado en el extranjero, pero la formación recibida fuera del país es, a la larga, más costosa y traumática, porque afecta los procesos mismos de la enseñanza y porque acrecienta la dependencia cultural que impide la generación de conocimientos adecuados para la realidad del país.

Como vemos, las brechas son profundas, casi abismales, y nos ayudan a entender el drama que vive un país que, a simple vista, no tiene expectativas de superación sino se da una verdadera revolución infraestructural en el campo educativo que arrastre los cambios en otros sectores.

En conclusión, basta citar un párrafo de la UNESCO, al respecto, que resume la tragedia de estos países:

«Estamos mal, y mientras no exista progreso técnico-científico no se puede aspirar a un mayor nivel económico. Vale la pena destacar los esfuerzos y logros 
en Ciencia y Tecnología por parte de Brasil, México, Argentina, Venezuela y, más modestamente, Colombia».

\section{Tercero}

Para concluir, nos referiremos a una propuesta académica que estamos liderando desde la Facultad de Derecho de la USCO, que es la Especialización en Derecho del Trabajo y la Seguridad Social.

En general, lo que llamaríamos la crisis del trabajo es un problema político resultado de una lucha que ha implicado el cambio del Estado proteccionista hacia el Estado neoliberal, la reestructuración productiva, la incapacidad de las organizaciones obreras de transformar sus formas de lucha, el reemplazo del conflicto obrero-patronal por la lucha de mercados, la apertura de mercados, la extensión de la subcontratación, la reducción del empleo público y la desregulación del mercado laboral, entre otros factores.

La Especialización busca contribuir, desde la educación formal en el nivel postuniversitario, al fortalecimiento de una verdadera cultura de la seguridad social y del derecho del trabajo, individual y colectivo, en la región Surcolombiana, en particular, y en el país, en general.

En consecuencia, y desde la perspectiva profesional esperamos que los especialistas en Derecho del Trabajo y Seguridad Social cumplan una importante función social, crítica y objetiva en cuanto a las relaciones laborales y las normas y principios que promueven la aplicación de la seguridad social. Por ello, nuestro especialista deberá estar en condiciones de emprender procesos comprensivos y administrativos de análisis, formulación y evaluación de proyectos y operaciones laborales relacionadas con el derecho al trabajo, la seguridad social y el bienestar de la ciudadanía, en general; tener la capacidad profesional suficiente para afrontar y plantear soluciones ,en asuntos sociales, jurídicos, económicos y financieros en materia del trabajo y la seguridad social, que desde el Derecho Laboral y desde los sistemas de salud, pensiones y riesgos profesionales, están regulados en Colombia, ateniéndose a los principios de solidaridad, universalidad $\mathrm{y}$ eficiencia.

Desde luego que aceptar la noción de trabajo desde esta óptica epistemológica, implica asumirlo desde una concepción distinta a la exclusivamente laboral o como producto que debe ser remunerado bajo normas y principios de aplicación obligatoria. Una concepción, como la que se propone aquí, reconoce dos aspectos esenciales: por un lado, la noción de trabajo como elemento clave de la productividad social e individual en el que el ser humano encuentra las oportunidades de su crecimiento paulatino y que le exige una mirada en constante 
retroalimentación. Por otro, el trabajo visto en su estrecha relación con el lenguaje y su interacción social, conformando la tríada de elementos que configuran lo que se ha denominada la construcción social de la realidad. Así, este enfoque nos aleja del concepto 'laboralista' del trabajo y nos conduce a una noción más dialéctica y comprensiva sobre la red que se teje en la configuración del ser humano dentro de un contexto social, político y jurídico determinado.

Al parecer, es urgente una revaloración de la dimensión del trabajo y la seguridad social, no sólo desde sus implicaciones jurídicas, sino también sociales. Así, el derecho a la seguridad social -consagrado en el artículo 22 de la Declaración Universal de Derechos Humanos- fue incorporado a nuestra Constitución Política, a favor de todas las personas y con el carácter de irrenunciable.

Con ello, se apunta a señalar la responsabilidad que le cabe al Estado en materia de dirección, control y coordinación del servicio público de la seguridad social, ya sea de modo directo o en convenio con el sector privado. Por otro lado, esta función pública de la seguridad social quedó determinada bajo los principios de solidaridad, universalidad y eficiencia, que son precisamente los que más han servido de controversia en las quejas, querellas y demandas interpuestas por usuarios del sistema en Colombia.

Lograr cambios cualitativos y cuantitativos de dicho sistema es posible en la medida en que se afronte, de modo objetivo y crítico, el actual estado en este campo como condición primaria para la postulación del cumplimiento de un servicio público social que acate los mandamientos jurídicos, sociales, financieros y económicos requeridos para la ampliación gradual y de calidad del sistema laboral.

Constitucionalmente, el tema nos remite a la Constitución de 1886 y sus reformas, en especial la dada en 1936, en la que el legislador aportó algunos elementos vitales de política social que tuvieron gran incidencia en el Estado liberal del momento. Dentro de estos elementos, cabe destacar la protección especial al trabajo y la asistencia pública como responsabilidad del Estado.

En el desarrollo de este mandato constitucional, progresivamente fueron apareciendo formas variadas de protección social para la población vinculada al trabajo de forma subordinada, como es el caso del régimen de previsión social de los trabajadores estatales, que surge a partir de la Ley 6 de 1945 y sus reformas; el régimen de los seguros sociales obligatorios, establecido con la Ley 90 de 1946; el régimen patronal de seguridad social, consagrado en el Código Sustantivo del Trabajo, en 1950, y ajustado por el Decreto Ley 2351, de 1965 y por la Ley 50 de 1990. 
De igual modo, se creó el régimen del subsidio familiar, en 1954 y, es más, en la misma Constitución de 1886, contemplaba, en su artículo 19, la función del Estado en cuanto a la asistencia pública, que llevó a la creación del Ministerio de Higiene (1947), el Plan Hospitalario Nacional (1963) y la reorganización del sistema de salud, en 1975.

Ha sido evidente que los esfuerzos han estado dirigidos a dar protección a grupos vulnerables, como ancianos, personas con limitaciones físicas, campesinos, etc. Ahora, la nueva Carta Política del país, asume la seguridad social desde tres argumentos relevantes:

-La generación de un Estado Social de Derecho.

-La concepción de Seguridad Social Integral.

- La estrategia de protección a grupos más vulnerables.

El primer precepto define al Estado colombiano como el llamado a «procurar el bienestar general y el mejoramiento de la calidad de vida de la población», en oposición a una concepción asistencialista, de beneficencia o caridad, que había primado hasta el momento; en síntesis, se desarrolla la idea de que los ciudadanos colombianos deben tener igualdad de oportunidades, no sólo ante la ley, sino ante la vida.

El segundo precepto, reconoce la obligatoriedad de ofrecer una seguridad social integral, que libere al ciudadano «de las contingencias que puedan crearle un estado de necesidad», convirtiendo la seguridad social en un derecho irrenunciable y garantizado, desde el Estado mismo.

El tercer precepto, reconoce la existencia de grupos más vulnerables que otros y, por ello, la necesidad de brindarles mecanismos de protección especial.

Desde esta visión, toda discusión en torno al derecho al trabajo y la seguridad social debe significar un avance grande para la sociedad colombiana, en aras del logro de los objetivos propuestos de bienestar general y mejoramiento de la calidad de vida, que creo que es lo que nos concita en este importante foro.

\section{Bibliografía}

BARBOSA DE ANDRADE, Néstor. Universidad Pública, Estado y Sociedad II. En Revista CRESAL / UNESCO, No. 3/96.

FACULTAD DE DERECHO. Estudio de Factibilidad para la creación de la Especialización en Derecho del Trabajo y la Seguridad Social. USCO, Neiva, 2003. 
GRAVETTO, Jorge. El Destino de la Universidad Latinoamericana. En Revista CRESAL / UNESCO No. 8/96.

MORIN, Edgar. Los siete saberes necesarios para la educación del futuro. UNESCO, París, 1999 http:// unesdoc.UNESCO.org/ulis/ged.html.

OROZCO SILVA, Luis Enrique. Financiamiento y Gestión de las Instituciones de Educación Superior en América Latina. En Revista CRESAL / UNESCO No. $4 / 96$.

ONU. Indicadores de Desarrollo Humano - 2003.

REVISTA UNIVERSITA 2000. Vol. 15, No. 4, 1991.

RODRÍGUEZ GÓMEZ, Roberto. El Porvenir de la Universidad Latinoamericana. En Revista CRESAL / UNESCO No. 3/96.V9. 